Case Report

\title{
Sitagliptin-Associated Pancreatitis: A Report of 4 Cases
}

\section{Aude Garin ${ }^{1}$, Florence Bouilloud ${ }^{2}$, Emmanuelle Masson ${ }^{3}$, Olivier Blétry ${ }^{4}$, Alfred Penfornis $^{4}$ and Pascal Hammel ${ }^{5}$}

${ }^{1}$ Intensive care unit, Centre hospitalier Victor Jousselin, Dreux Cedex

2,4 Internal Medicine department, Diabetology, Hôpital Foch, Suresnes

${ }^{3}$ Laboratory of Molecular Genetics and Histocompatibility, Hôpital Morvan, Brest

${ }^{4}$ Diabetology Endocrinoly Unit, Hôpital J. Minjoz, Besançon

${ }^{5}$ Gastroenterology-Pancreatology Department, Hôpital Beaujon, Clichy

Correspondence should be addressed to: Aude Garin; audegarin@hotmail.fr

Received date: 16 April 2014; Accepted date: 14 August 2014; Published date: 28 December 2015

Academic Editor: Martine Vaxillaire

Copyright (C) 2015. Aude Garin, Florence Bouilloud, Emmanuelle Masson, Olivier Blétry, Alfred Penfornis and Pascal Hammel. Distributed under Creative Commons CC-BY 4.0

\begin{abstract}
Objective: Sitagliptin is a new oral glucose-lowering medication that acts via the incretin hormone system. The most common side-effects are headache and pharyngitis. Acute pancreatitis is rarely described in literature. Research design and Methods: We report four cases of pancreatitis that developed in patients after 9 days to 18 months of treatment with sitagliptin. Result: The origin of acute pancreatitis has been attributed to the administration of sitagliptin after a thorough analysis of the different possible etiologies with different accountabilities defined by Delcenserie R. (2001) as the time of onset criteria, the existence or not of other metabolic, toxic or genetic causes.Conclusion: Acute pancreatitis in patients receiving sitagliptin seems to be rare but physicians should be aware of this possibility.
\end{abstract}

Keywords: Sitagliptin, DPP-4 inhibitor, acute pancreatitis

Cite this Article as: Aude Garin, Florence Bouilloud, Emmanuelle Masson, Olivier Blétry, Alfred Penfornis and Pascal Hammel (2015), "Sitagliptin-Associated Pancreatitis: A Report of 4 Cases" International Journal of Case Reports in Medicine, Vol. 2015 (2015), Article ID 198809, DOI: 10.5171/2015.198809 


\section{Abbreviations:}

DPP: dipeptidyl peptidase

HbA1c: glycosylated hemoglobin

PRSS1: protease serine 1 (trypsin 1)

SPINK1: serine proteinase inhibitor Kazal type 1

CFTR: cystic fibrosis transmembrane conductance regulator

$\mathrm{N}$ : normal upper value

\section{Introduction}

Several large clinical studies have confirmed the efficacy and safety of the dipeptidyl peptidase (DPP)-4 inhibitor sitagliptin for the management of type 2 diabetes. Pharmacological inhibition of DPP-4 is an alternate approach to increase circulating concentrations of endogenous active GLP-1 and thus glucose-dependent insulin secretion. The most frequent adverse events with sitagliptin include nausea, an increased risk of rhinopharyngitis and headache. Although pancreatitis, due to sitagliptin administration, has been reported, there are only a few documented cases [Egan AG (2014), Engel S. S. and Golm G. T. (2010), Engel S. S. and Round E. (2010), Food and Drug Administration (2009), Garg R. and Chen W. (2010), Garg R. and Hussey C. (2010), Girgis C. M. (2011), Ligueros-Saylan M. (2010) and Monami M. (2014)]. Sitagliptin therapy has been shown to result in increased pancreatic duct replication, acinar to ductal metaplasia and less frequently, acute pancreatitis in a rat model of type 2 diabetes [Matveyenko A. V. (2009)].

We report four patients followed in a single hospital (Foch, Suresnes, France) who developed acute pancreatitis, which was probably sitagliptin-induced.

\section{History and examination}

\section{Case 1}

A 58 year-old woman presented with the following medical history: thyrotropic pituitary macroadenoma with secondary gonadotropic and somatotropic failure, hypertriglyceridemia treated with fenofibrate and post smoking chronic obstructive pulmonary disease. The patient had also undergone a cholecystectomy for biliary colic in 2004 and removal of a residual stone of the main bile duct in 2008 . In July 2010, uncomplicated type 2 diabetes mellitus was diagnosed during a routine hospital stay. The glycosylated hemoglobin (HbA1c) level was 7.9\%. The treatment was begun with $60 \mathrm{mg}$ gliclazide and $100 \mathrm{mg}$ sitagliptin daily. In January 2011, the patient was admitted to the emergency unit due to radiating epigastric pain with an occlusive syndrome. The lipase serum level was 2577 UI/L (normal upper value: 227 UI/L). Liver tests were normal except for a slight and stable increase in gamma glutamyl transpeptidase at $2 \mathrm{~N}$. There were no biological signs of severity (Ranson $=0$ ). To obtain a diagnosis, an abdominal computed tomography (CT) scan was performed and showed fatty infiltration of the pararenal area behind the tail of the pancreas (Balthazar 2 points). There was no biliary lithiasis or pancreatic duct abnormalities on MRI. Patient characteristics (origin, body mass index) and the risk factors for pancreatitis are shown in tables 1 and 2 including alcohol consumption, biliary lithiasis, pancreatic duct abnormalities or calcifications (on CT scan and MRI), metabolic abnormalities (lipids, calcemia), pancreatotoxic medication intake, family history and genetic abnormalities (protease serine 1 (trypsin 1) (PRSS1), serine proteinase inhibitor Kazal type 1 (SPINK1) or the cystic fibrosis transmembrane conductance regulator (CFTR) gene (the 30 most frequent mutations were searched for using the CFTR gene kit Elucigen CF30, Tepnel. Diagnostics, Oxon, UK)). There was no evidence of autoimmune disease. The patient was treated for symptoms (analgesics, fasting). The course was 
favorable and the patient was discharged three days later. Sitagliptin was stopped and replaced by insulin and metformin. There was no relapse of pancreatitis at 10 months of follow-up (table 3).

\section{Case 2}

A 69 year-old man presented with a history of hypertension and moderate hypertriglyceridemia (3 g/L) treated with atorvastatin. He had had type 2 diabetes mellitus for 10 years and was initially treated with metformin which was later combined with glibenclamide. Diabetes was complicated by diabetic retinopathy, neuropathy of the lower limbs, and nephropathy of a mixed diabetic and hypertensive origin (creatinine baseline 140 $\mu \mathrm{mol} / \mathrm{L}$ ). Patient characteristics (origin, body mass index) and the risk factors for pancreatitis are shown in tables 1 and 2 . Because diabetes was not sufficiently controlled, triple therapy with metformin $1000 \mathrm{mg}$ tid, sitagliptin $100 \mathrm{mg}$ once a day and glimepiride was begun in September 2009. Nine days later, the patient was admitted for abdominal pain, nausea and vomiting. Lipase serum levels were $9900 \mathrm{UI} / \mathrm{l}$ $(\mathrm{N}<227 \mathrm{UI} / \mathrm{L})$. Liver tests were normal, the alkaline reserve was $13 \mathrm{mmol} / \mathrm{L}$ and serum creatinine was $1700 \mu \mathrm{mol} / \mathrm{L}$. Unenhanced abdominal CT scan showed a minor peripancreatic infiltration without any collection (Balthazar 2 points). There was no biliary lithiasis or pancreatic ducts abnormalities on ultrasound or MRI. There was no evidence of an autoimmune disease. There were no biochemical signs of severity (Ranson 0). Sitagliptin was then stopped. Clinical symptoms improved, lipase levels rapidly normalized, and the patient was discharged after five days. There was no relapse of pancreatitis after 25 months of follow-up (table 3).

\section{Case 3}

A 49 year-old woman presented who had had type 2 diabetes since 2003 and had initially been treated with glibenclamide, acarbose and metformin, as well as atorvastatin for dyslipidemia. Diabetes mellitus was complicated by diabetic retinopathy, neuropathy and lower limb arterial occlusive disease. In July 2009, a combination of sitagliptin $100 \mathrm{mg}$ once a day and glargine insulin at bedtime was begun because diabetes was not sufficiently controlled (HbA1c at 10.6\%). Patient characteristics (origin, body mass index) and the risk factors for pancreatitis are shown in tables 1 and 2 . In October 2010, the patient was admitted after two days of epigastric pain in the upper-right quadrant. Serum lipase levels were $884 \mathrm{UI} / \mathrm{L}(\mathrm{N}$ <227) and liver tests were normal. The next day, a contrast enhanced CT scan showed enlargement of the pancreatic head and infiltration of the peripancreatic fat without parenchyma necrosis (Balthazar 2 points). There was no biliary lithiasis or pancreatic ducts abnormalities on ultrasound or MRI. There were no calculi in the common bile duct. There was no evidence of autoimmune disease. The patient was treated for symptoms and was discharged five days later, after pain had disappeared and lipase levels had normalized. Sitagliptin was then stopped. There was no gallbladder lithiasis or other biliopancreatic abnormalities on endoscopic ultrasound one month later. There was no relapse of pancreatitis after 11 months of follow-up (table 3).

\section{Case 4}

A 52 year-old man presented with the following medical history: hypertension and uncomplicated type 2 diabetes diagnosed in 2004 and treated with metformin. The patient characteristics (origin, body mass index) and the risk factors for pancreatitis are shown in table 1 and 2 . The patient was prescribed sitagliptin $100 \mathrm{mg}$ per day in December 2008, and glycemia was controlled with $\mathrm{HbA} 1 \mathrm{c}$ at $7.4 \%$. In June 2010, the patient was admitted with epigastric pain, nausea and vomiting. Lipase serum levels were $25,559$ UI / L ( $<227)$ and liver tests were normal. A contrast enhanced CT scan showed infiltration of the peripancreatic fat and left pre-renal necrosis (Balthazar 3 points). Sitagliptin was stopped. After 9-days in the intensive care unit with a simplified acute 
physiology score (SAPS) then 16 days in a surgery unit, he was referred to the diabetes unit for a basal-bolus insulin regimen with lispro and glargine insulins. There was no biliary lithiasis or pancreatic duct abnormalities on ultrasound or MRI. There was no evidence of autoimmune disease. There was no relapse of pancreatitis after 16 months of follow-up (table 3).

\section{Investigation}

Three of these four cases of pancreatitis can be reasonably attributed to sitagliptin, while case number 4 is less certain (table 4 ).

Because of the risk of a relapse of sometimes severe pancreatitis if there is a drug readministration and because several antidiabetic treatments are available, we did not do a sitagliptin rechallenge in any of the patients. Delays suggested or were compatible with sitagliptin-induced pancreatitis in two patients, while they were less suggestive in the two others. Indeed, delays of 15 and 18 months (cases ${ }^{\circ} 3$ and 4) were less evocative while not excluding the responsibility of sitagliptin. (table 3). Patients had no significant chronic alcohol consumption and only one was addicted to tobacco. Biliary pancreatitis or hyperlipidemia was unlikely although patients were $>45$ years old and BMI > 30 $\mathrm{kg} / \mathrm{m} 2$ in two of them. The effect of a high $\mathrm{BMI}$ on the risk of pancreatitis is limited and equivalent to a 1.2-fold increased risk per five units of BMI [Blomgren K. B. (2002)]. All patients had normal serum triglyceride levels at diagnosis. Liver tests were normal at admission and imaging tests did not suggest biliary lithiasis. Calcemia was normal in all cases. There were no duct/parenchyma abnormalities on either CT scan or MRI. No other pancreatotoxic drugs had been recently added and there was no history of abdominal trauma or radiotherapy. Analysis of genetic factors is still rare in the reported cases of drug-associated pancreatitis. It is interesting to note that two of the four patients had a genetic abnormality in the CFTR or SPINK1 gene (table 2). Minor alterations in these genes can be found in the general population
(CFTR $\sim 5 \%$ [Sharer N. (1998)] and SPINK1 1-2 \% [Pfützer R. H. (2001)]) and the latter could have been a co-factor for pancreatitis in these two patients. Autoimmune pancreatitis is improbable because there was no context for this and no features on imaging results. Although IgG4 serum levels were not measured, they are normal in up to $80 \%$ of cases of type 1 autoimmune pancreatitis in Europe [Maire F. (2011)]. Finally, there was no other potential cause of acute/chronic pancreatitis.

Identifying drug-induced pancreatitis in patients with type 2 diabetes mellitus can be difficult due to confounding factors, such as patients receiving various potentially pancreatotoxic medications: fenofibrate, statins, angiotensin receptor antagonist and angiotensin-converting enzyme inhibitors. Likewise some other oral hypoglycemic agents as GLP1, metformin and glyburide may cause pancreatitis [Cohen (2013), Fimognari (2006)]. However, only two of these patients were receiving this type of drug and had been for more than five years. Thus, their responsibility seems unlikely.

Preclinical studies of sitagliptin toxicity during drug development program showed the potential occurrence of pancreatitis in animal testing, what opposes a recent study in which smiled were exposed to sitagliptin during a year, without increased risk of pancreatitis [Aston-Mourney K. (2013)]. In contrast, this has been poorly documented in humans. One case was reported in a 53-year old woman who was treated for type 2 diabetes with metformin $1000 \mathrm{mg} \times 2$ /day and gliclazide $160 \mathrm{mg} /$ day for 6 years [Garg R. (2010)]. She had also taken orlistat to lose weight, which may result in rare cases of acute pancreatitis in the first days it is taken. However, the patient had been taking it for more than eight months with no pancreatic pain. Finally, imputability was not evident. Similarly, a case of acute pancreatitis was reported in a patient receiving vildagliptin [Girgis C. M. (2011)]. This 61-year oldwoman had had type 2 diabetes mellitus for five years. She has been receiving sitagliptin for six months and then switched to 
vitagliptin five weeks before the diagnosis of acute pancreatitis. Because of the absence of any obvious secondary cause for pancreatitis and the temporal sequence of events, this case suggests a causal relationship between the initiation of vildagliptin and the development of pancreatitis.

Likewise, the FDA lists 88 post-marketing cases of acute pancreatitis, including two cases of "hemorrhagic" or "necrotizing" pancreatitis in patients using sitagliptin between October 2006 and February 2009 [Food and Drug Administration. (2009)]. The clinical symptoms were usual (pain, nausea and vomiting). Twenty one percent of the cases occurred within the first 30 days of the treatment with sitagliptin. The course was favorable in $53 \%$ after the drug was withdrawn. Half of the patients had other risk factors such as type 2 diabetes mellitus, obesity and hyperlipidemia. A recent report analyzing the FDA database of reported adverse events associated with the use of sitagliptin from 2004 - 2009, has suggested that pancreatitis may be $>6$-fold more frequent with this drug compared to other antidiabetic therapies (OR: 6,86 [IC95\%: 4.68 to 10.2], p-value < 10-16). The authors state that obesity, which was not recorded in this database, type 2 diabetes and tobacco consumption may have been confounding factors for the risk of pancreatitis. Finally, the limitations of the FDA adverse events report database are well-known including incomplete data and reporting biases [Elashoff M. (2011)].

Similarly, in 2012, the French regional pharmacovigilance centers have reported eight cases of acute pancreatitis in patients receiving sitagliptin. There were three women and five men aged 52 - 81 years old who had been taking sitagliptin for between nine days and one year. All of them had a favorable course after the drug was withdrawn. One patient had gallbladder lithiasis without bile duct enlargement; there were no available data in the remaining patients (unpublished data).
In a series of $>8000$ patients receiving vildagliptin compared to a control group of 6210 patients receiving other oral antidiabetic medications, there was no evidence of an increased risk of pancreatitis. $\mathrm{OR}<1(0.10-0.24 \%$ in the vildagliptin groups vs $0.17 \%$ ). It did not result in a greater incidence of acute pancreatitis when used for periods of between 12 weeks - 2 years. A previous analysis pooled 12 double-blind randomized studies of up to 2 years in patients with type 2 diabetes mellitus including 6139 patients treated with either sitagliptin $(n=3415)$ or a comparative agent (placebo or other oral antidiabetic drugs, $\mathrm{n}=$ 2724). There was no difference in the incidence of pancreatitis between the two groups [Engel S. S. and Golm G. T. (2010)]. Moreover, Ligueros-Saylan et al. (2010) have reported a $0.13 \%$ incidence of acute pancreatitis with vildagliptin and $0.24 \%$ in controls; and Garg R., Chen W. and Pendergrass M. (2010) , an incidence of 0,4\% of acute pancreatitis with sitagliptin (RR 2,1, CI95\% : 1.7-2.5).

Finally, several meta-analyzes have reported no increased risk of acute pancreatitis [Li L. (2014)], especially two of them, the first safety of sitagliptin with 19 double-blind randomized studies (10 246 patients with type 2 diabetes) and the second with 109 studies taking into account all DDP4 inhibitors against comparators, [Engel S. S. and Round E. (2010), Matveyenko A. V. (2009)]. Nevertheless, we note that the issue of meta-analyses is often hampered by the introduction of bias or confounding factors related to the heterogeneity of the different sub-groups of patients.

Finally, we wish to clarify that this analysis was performed independently of the FDA.

\section{Conclusions}

Acute pancreatitis in patients receiving sitagliptin seems to be rare but physicians should be aware of this possibility and be cautious about the monitoring of long-term treatment. 


\section{Acknowledgments}

Dr. A. Garin is the guarantor of this work and, as such, had full access to all of the data in the study and takes responsibility for the integrity of the data and the accuracy of the data analysis.

\section{References}

1. Aston-Mourney K., Subramanian S. L., Zraika S., Samarasekera T., Meier D. T., Goldstein L. C. and Hull R. L. (2013) "One year of sitagliptin treatment protects against islet amyloid-associated $\beta$-cell loss and does not induce pancreatitis or pancreatic neoplasia in mice. Am J Physiol Endocrinol Metab, 305: E475-84.

2. Blomgren K. B., Sundström A., Steineck G. and Wiholm B. E. (2002) "Obesity and treatment of diabetes with glyburide may both be risk factors for acute pancreatitis". Diabetes Care, 25:298-302.

3. Cohen, Deborah (2013) "Two drugs for type 2 diabetes seem to raise risk of acute pancreatitis". BMJ, 346:2.

4. Fimognari F.L., Corsonello A., Pastorell R. and Antonelli-Incalzi R. (2006) "Metformininduced pancreatitis: A possible adverse drug effect during acute renal failure". Diabetes Care., 29(5):1183.

5. Delcenserie R. (2001) "What are the criteria for imputation of drug-induced pancreatitis ?" . Gastroenterol Clin Biol., 25(1 Suppl):1S18-21.

6. Egan AG, Blind E, Dunder K, de Graeff PA, Hummer BT, Bourcier T, Rosebraugh C. (2014) "Pancreatic safety of incretin-based drugs--FDA and EMA assessment". $N$ Engl J Med., 27;370:794-7.

7. Elashoff M., Matveyenko A. V., Gier B., Elashoff R. and Butler P. C. (2011) "Increased incidence of pancreatitis and cancer among patients given glucagon like peptide- 1 based therapy" Gastroenterology, 141:150-6.
8. Engel S. S., Williams-Herman D. E., Golm G. T., Clay R. J., Machotka S. V., Kaufman K. D. and Goldstein B. J. (2010) "Sitagliptin: review of preclinical and clinical data regarding incidence of pancreatitis". Int J Clin Pract, 64:984-90

9. Engel S. S., Williams-Herman D. E., Round E., Johnson J., Guo H., Musser B. J., Davies M. J., Golm G. T., Kaufman K. D. and Goldstein B. J. (2010) "Safety and tolerability of sitagliptin in clinical studies: a pooled analysis of data from 10,246 patients with type 2 diabetes" BMC Endocrine Disorders, 10:7.

10.Food and Drug Administration. September 25, (2009). Information for healthcare professionals-acute pancreatitis and sitagliptin (marketed as Januvia and Janumet).

11.Garg R., Chen W. and Pendergrass M. (2010) "Acute pancreatitis in type 2 diabetes treated with exenatide or sitagliptin: a retrospective observational pharmacy claims analysis" Diabetes Care, 33:2349-54.

12.Garg R., Hussey C. and Ibrahim S. (2010) "Pancreatitis associated with the use of sitagliptin and orlistat combination: a case report" Diabetic Medicine, 27:485-486.

13.Girgis C. M. and Champion B. L. (2011) "Vildagliptin-induced acute pancreatitis" Endocrine Practice, 17:48-50.

14.Ligueros-Saylan M., Foley J. E., Schweizer A., Couturier A. and Kothny W. (2010) "An assessment of adverse effects of vildagliptin versus comparators on the liver, the pancreas, the immune system, the skin and in patients with impaired renal function from a large pooled database of Phase II and III clinical trials" Diabetes, Obesity and Metabolism, 12:495-509.

15.Li L., Shen J., Bala M.M., Busse J.W., Ebrahim S., Vandvik P.O., Rios L.P., Malaga G., Wong E., Sohani Z., Guyatt G.H., Sun X. (2014).“Incretin treatment and risk of pancreatitis in patients with type 2 diabetes mellitus: systematic review and meta- 
analysis of randomised and non-randomised studies". BMJ. 15;348:2366.

16. Maire F., Le Baleur Y., Rebours V., Vullierme M. P., Couvelard A., Voitot H., Sauvanet A., Hentic O., Lévy P., Ruszniewski P. and Hammel P. (2011) "Outcome of patients with type 1 or 2 autoimmune pancreatitis". Am J Gastroenterol, 106:151-6.

17. Matveyenko A. V., Dry S., Cox H. I., Moshtaghian A., Gurlo T., Galasso R., Butler A. E. and Butler P. C. (2009) "Beneficial endocrine but adverse exocrine effects of sitagliptin in the human islet amyloid polypeptide transgenic rat model of type 2 diabetes: interactions with metformin". Diabetes, 58:1604-15.
18. Meier JJ, Nauck MA. (2014) "Risk of pancreatitis in patients treated with incretinbased therapies". Diabetologia.;57(7):1320-4

19.Monami M., Dicembrini I. and Mannucci E. (2014) "Dipeptidyl peptidase-4 inhibitors and pancreatitis risk: a meta-analysis of randomized clinical trials" Diabetes, Obesity and Metabolism, 16:48-56.

20.Pfützer R. H. , Barmada M. M. and Whitcomb D. C. (2001) "Mutations of the pancreatic secretory trypsin inhibitor gene in idiopathic chronic pancreatitis" Gastroenterology, 120:1063-4.

21.Sharer N., Schwarz M., Malone G., Howarth A., Painter J. and Super M. (1998) "Mutations of the cystic fibrosis gene in patients with chronic pancreatitis" N Engl J Med, 339:64552. 